\title{
Productividad agropecuaria: reducción de la brecha productiva entre el Brasil y los Estados Unidos de América
}

\section{José Eustáquio Ribeiro Vieira Filho y Armando Fornazier}

RESUMEN

Desde los años setenta, la agricultura del Brasil ha experimentado grandes transformaciones destacándose en la producción agrícola mundial. Para verificar la heterogeneidad estructural en la agricultura brasileña y estadounidense, aquí se estudia la productividad total de los factores (PTF) y se observa su aumento en ambas economías, principalmente mediante tecnologías que permiten ahorrar tierra y trabajo. Aunque en el Brasil se registraron mayores tasas de crecimiento que hicieron posible reducir la brecha productiva en comparación con los Estados Unidos de América, esto no implica que la productividad brasileña sea superior. La estructura productiva presenta particularidades en los dos casos. Las diferencias de productividad no solo se observan entre países, sino también dentro de un mismo país debido a diversos factores: clima, tecnología y aprendizaje en el proceso de producción. La eficiencia en el uso de los recursos, que permite producir más con menos insumos, aumentó en ambos países. Regionales, Urbanos y Ambientales (DIRUR) del Instituto de Investigación Económica Aplicada (IPEA). jose.vieira@ipea.gov.br

Armando Fornazier es Docente en la Facultad de Agronomía y Medicina Veterinaria (Fav) de la Universidad de Brasilia (UnB), Brasil. armandouenf@yahoo.com.br 


\section{I}

\section{Introducción}

En las últimas décadas, la agricultura brasileña ha experimentado grandes transformaciones que condujeron a la modernización, la incorporación de tecnología y el aumento de la productividad del sector. El Brasil pasó a ser considerado un gran productor mundial de alimentos y biocombustibles, junto con otros países como los Estados Unidos de América. Sin embargo, algunos establecimientos productivos presentan bajo contenido tecnológico, reducida productividad y están todavía distantes de los modelos de desarrollo modernos ${ }^{1}$. Incluso hay productores que, debido a la escasa producción, muchas veces quedan excluidos de los mercados y en algunos casos en situación de pobreza rural. Esto refleja la heterogeneidad de la agricultura brasileña.

La productividad del trabajo, calculada por medio de la fracción entre el valor agregado y la población ocupada de cada establecimiento, es la variable de referencia que dimensiona la heterogeneidad estructural en los estudios de la Comisión Económica para América Latina y el Caribe (CEPAL) (Pinto, 1970; Nohlen y Sturm, 1982; Sunkel e Infante, 2009; CEPAL, 2010; Vieira Filho, Santos y Fornazier, 2013). La heterogeneidad puede medirse por la variabilidad de la productividad del trabajo entre países (aquí denominada brecha productiva internacional), entre sectores (industria, servicios y agricultura) y dentro de la propia agricultura (comparación de agentes productivos y cultivos específicos).

El objetivo de la comparación consiste en mostrar los modelos de competitividad externa de la agricultura brasileña mediante la reducción de la brecha productiva internacional y establecer el desarrollo dual que persiste en la economía e impide la inclusión productiva de los segmentos marginados del proceso de modernización tecnológica.

De acuerdo con Fornazier y Vieira Filho (2012); Vieira Filho, Santos y Fornazier (2013), y Vieira Filho

\footnotetext{
${ }^{1}$ Véase un extenso análisis de los resultados del Censo Agropecuario de 2006 en Gasques y otros (2010). Ese estudio proporciona una visión general de la transformación más reciente en la agricultura brasileña. Buainain y otros (2014) promueven un amplio debate sobre las transformaciones tecnológicas de la agricultura brasileña en el período de 1960 a 2014, cuando el Brasil pasó de ser importador neto de alimentos a exportador y relevante productor en el escenario mundial (a partir de la década de 1980).
}

(2013), existe una enorme heterogeneidad productiva en la producción agropecuaria, y en particular en el caso brasileño. Mientras algunos productores emplean técnicas modernas para incrementar la productividad, otros solo pueden adoptar técnicas con menor intensidad tecnológica debido a las dificultades de acceso a las tecnologías más modernas, o incluso a los obstáculos relacionados con el proceso de adaptación.

La combinación de los factores de producción se expande a medida que la agricultura se moderniza, pues los productores tienen la opción de adoptar tecnologías que les permitan ahorrar algunos recursos (como la tierra o el trabajo) y sustituirlos por otros (como por ejemplo, una mayor cantidad de capital). Para modificar la producción, las propias políticas agrícolas -incluidas las de crédito (muchas veces subsidiado) - incrementaron la capitalización de la agricultura mediante la promoción de relaciones con otros sectores - como el de insumos $\mathrm{y}$ el formado por las agroindustrias, el comercio y los supermercados, entre otros ligados a la producción-, la definición de modelos e incluso la promoción de financiamientos.

El desarrollo no tiene lugar de manera homogénea entre países y regiones, o incluso dentro de un mismo sector o actividad. A lo largo del proceso se crean diferencias en los ambientes económico, social, político, tecnológico y cultural. La discusión teórica de las diferencias de desarrollo entre los países surgió en el marco de la CEPAL en la década de 1950, cuando se abordaron principalmente las disimilitudes entre los centros desarrollados y las periferias en desarrollo (Nohlen y Sturm, 1982).

Pinto (1970) ya había definido la heterogeneidad estructural como un fenómeno visible en los países en desarrollo, en particular en América Latina. Se señala que la productividad de la agricultura moderna sería unas 14 veces mayor que la del sector tradicional. No todos los productores lograron modernizarse. Existen diferencias regionales significativas que pueden explicar la convivencia de sectores modernos y rezagados.

Las diferencias sectoriales, o incluso dentro de una misma actividad económica, obedecen a problemas de acceso a los recursos financieros para la modernización y al nivel de conocimiento de los actores sociales (capital social), que influyen en la capacidad de promover cambios. 
Algunos factores culturales, climáticos y regionales también pueden alterar la producción local y aumentar las disparidades entre las regiones.

Las diferencias sectoriales y dentro de un mismo sector forman parte de la historia del Brasil. De acuerdo con Fornazier y Vieira Filho (2012) y Vieira Filho (2013), la heterogeneidad se presenta tanto a nivel de la producción como de la región. La coexistencia de una agricultura moderna, orientada al mercado externo, con otra dedicada casi exclusivamente a la subsistencia de las familias del campo y al abastecimiento de pequeños mercados locales es histórica. Independientemente del tamaño, la agricultura más productiva es comparable con la agricultura de la frontera tecnológica mundial.

En el campo económico, es posible utilizar algunos indicadores para examinar la heterogeneidad estructural. Nohlen y Sturm (1982) señalan, por ejemplo, que la productividad puede ser un indicador para delimitar investigaciones y mediciones en la economía. La creación de índices permite verificar las disparidades existentes y la trayectoria de los modelos de desarrollo de determinadas actividades. Según la CEPAL (2010, pág. 24): "La convergencia productiva implica cerrar las brechas de productividad con los países más competitivos, pero también reducir la heterogeneidad estructural interna. Estas brechas relativas a la productividad y la sociedad tienen sus mapas, es decir, se plasman en la segmentación territorial y, a la vez, se nutren de ella".

Para comparar la actividad agropecuaria entre los países, es importante estudiar los aspectos históricos de su desarrollo mediante el análisis de los factores que con el tiempo dieron lugar a una diferenciación. Las disparidades con respecto a los sectores o países más modernos pueden disminuir o aumentar con los años, a medida que en los más avanzados, donde existe mayor nivel de conocimiento acumulado, mejora la combinación de factores. En consecuencia, la evaluación de la productividad es importante para determinar las diferencias tecnológicas y productivas.

El objetivo de este estudio consiste en establecer si el desarrollo de la economía brasileña se aproxima a los incrementos de productividad alcanzados en la frontera tecnológica de la producción agropecuaria, y analizar la heterogeneidad estructural en ese sector. Con fines comparativos, se analiza el sector agropecuario brasileño en relación con la economía estadounidense, que en este trabajo se toma como referencia de frontera tecnológica. Se investiga la evolución de las brechas productivas entre el Brasil y los Estados Unidos de América a lo largo del tiempo, específicamente las diferencias en la productividad total de los factores (PTF), y se elabora un cuadro comparativo de los dos países.

Los resultados indican que, pese a la convergencia de la productividad agrícola del Brasil con la estadounidense en las últimas cuatro décadas, todavía existe una considerable heterogeneidad productiva en el sector agropecuario brasileño. Por una parte, se observa la convergencia de la PTF de los dos países y, por otra, se constata que la heterogeneidad intrarregional en el sector agropecuario del Brasil es aún muy elevada.

El presente artículo se organiza de la siguiente manera: en la sección II se aborda el desarrollo agropecuario en ambos países. En la sección III se presenta el método de análisis que consiste en la medición de la productividad total de los factores (PTF). En la sección IV se lleva a cabo una comparación de la PTF entre el Brasil y los Estados Unidos de América y en la sección V se ofrecen las consideraciones finales.

\section{II}

\section{EI desarrollo agropecuario en el Brasil}

\section{y los Estados Unidos de América}

La actividad agropecuaria constituye un sector dinámico de la economía brasileña, que realiza una importante contribución al producto interno bruto (PIB) y a las exportaciones del país, genera empleo y produce alimentos y energía (Vieira Filho, 2014).

Sin embargo, se trata de un sector heterogéneo desde su modernización, según la descripción de Paiva (1971), que delineó el proceso modernizador de la agricultura y la dualidad o multiplicidad tecnológica. En algunas regiones más desarrolladas desde el punto de vista económico, el porcentaje de agricultores modernos es siempre muy elevado con respecto a las demás categorías. En contrapartida, la situación se invierte en las regiones denominadas "atrasadas" y el porcentaje de agricultores 
tradicionales se vuelve predominante o incluso absoluto. No obstante, entre esos extremos hay regiones en desarrollo en las que el grado de modernización asume los valores más diversos. Además de las diferencias entre regiones, Paiva (1971) también destaca las disparidades en el grado de modernización entre productos en una misma región.

La modernización cambia las relaciones de producción debido al aumento del capital y de los vínculos de la agricultura con otros sectores, como la industria de transformación o la agroindustria ${ }^{2}$. Se define como el proceso de transformación en la base técnica de la producción agropecuaria en el período de posguerra, a partir de las importaciones de tractores y fertilizantes con miras a aumentar la productividad (Graziano da Silva, 1996, pág. 19).

Las políticas públicas, en particular el crédito rural, también brindaron a muchos productores la oportunidad de utilizar recursos más modernos en la agricultura. Sin embargo, no todos tuvieron acceso a esos recursos. El cambio en la base técnica acrecienta la necesidad de inversión agrícola y, de ese modo, el crédito se vuelve esencial para acceder a las tecnologías más modernas. Ciprandi y Fert Neto (1996) subrayan que, en el caso brasileño, las políticas públicas de incentivo a la modernización beneficiaron sobre todo a las propiedades grandes y medianas.

Otros factores, como la apertura comercial de los mercados, hicieron que muchos productores mejoraran sus técnicas de producción y gestión para poder competir con los productos importados. Coura, Figueiredo y Santos (2006) observaron que la eficiencia técnica de algunos cultivos (como algodón, arroz, frijoles, maíz y soja) registró una variación positiva en el período posterior a la apertura de la economía brasileña, especialmente a partir de la implementación del real en 1994. Estos autores destacan que la agricultura de São Paulo reaccionó a la mayor competencia externa provocada por la apertura, combinada con la valorización de la moneda nacional y el aumento de la productividad. Los beneficios no se distribuyeron de manera uniforme entre las distintas regiones y quedó en evidencia la obtención de mayores ganancias en las regiones que cuentan con más apoyo de los servicios de asistencia técnica y extensión rural.

Además de la liberalización comercial, la gran demanda impulsada por el crecimiento de muchos países en desarrollo propició una mayor producción de alimentos y materias primas y constituyó una oportunidad para que los productores buscaran más eficiencia y

2 Sobre la Revolución Verde, véanse Chianca (2004) y Fuck y Bonacelli (2007). obtuvieran ganancias en esos mercados "emergentes". De acuerdo con Wilkinson (2010), la demanda de los países emergentes ha dado nuevo aliento al ciclo de vida de los principales productos básicos. En los últimos años también ha aumentado la participación del sector financiero en los agronegocios. En el caso de los granos y las oleaginosas, se han elaborado nuevos documentos para viabilizar el mercado futuro y garantizar el financiamiento anticipado. Se observa asimismo una mayor participación de los grandes comerciantes en el financiamiento de la cosecha.

Algunos cultivos brasileños presentan patrones de productividad similares a los de los países que constituyen referencias mundiales. Gasques, Bastos y Bacchi (2008) afirman que la PTF de la agricultura brasileña (que es una relación entre la suma de todos los productos y la suma de todos los insumos) ha registrado tasas elevadas y crecientes en los últimos 30 años. Según estos autores, el promedio de crecimiento anual en ese período fue del $2,51 \%$, porcentaje superior al observado por Ball (2006) en los Estados Unidos de América.

La industria agropecuaria estadounidense se caracteriza por su elevada productividad, la adopción de tecnologías y extensas áreas de producción, que hacen de ese país un gran productor agrícola mundial. En 2010, por ejemplo, fue el mayor productor de maíz, seguido por China y el Brasil, y el mayor productor de soja, seguido por el Brasil ${ }^{3}$.

Los altos índices de productividad alcanzados por el sector agropecuario estadounidense se han convertido en puntos de referencia para muchos países. Sin embargo, la adopción de tecnologías y la modernización son el resultado de períodos anteriores con respecto al Brasil. Esto ocurre sobre todo en el caso de las tecnologías de ahorro de mano de obra, como señalan Hayami y Ruttan (1988) al comparar a los Estados Unidos de América -donde se adoptaron tecnologías para ahorrar trabajo debido a la escasez de mano de obra en el territoriocon el Japón, donde se introdujeron tecnologías que permitían el ahorro de tierra, por ser esta el recurso más escaso en ese país ${ }^{4}$.

En conformidad con Fuglie, MacDonald y Ball (2007), el trabajo en el sector agropecuario estadounidense disminuyó rápidamente en las décadas de 1950, 1960 y

\footnotetext{
${ }^{3}$ Véanse más detalles en FAO (2012).

${ }^{4}$ Véase una revisión crítica de la literatura y el papel de las economías de aprendizaje en Vieira Filho y Silveira (2012). Los autores trabajan con el modelo de difusión tecnológica, el dualismo productivo y la innovación inducida, incorporando al debate elementos de la competencia tecnológica (Vieira Filho, Campos y Ferreira, 2005) y del aprendizaje de los agentes (Vieira Filho y Silveira, 2011).
} 
1970, debido al aumento de los insumos (nuevas máquinas agrícolas e insumos químicos mejorados) y, sobre todo, a la reducción del trabajo agrícola, pues el incremento del costo del trabajo incentivó la adopción de tecnologías por parte de los agricultores. La transformación comenzó con el mejoramiento de la calidad de los insumos, entre ellos máquinas y productos químicos con nuevas formas de aplicación, que en muchos casos disminuían la carga química por hectárea sin comprometer el rendimiento del cultivo. Por otra parte, la mayor escala e integración entre los productores rurales, los proveedores de insumos y los procesadores han mejorado, entre otras áreas, las prácticas de producción animal.

Las innovaciones, sobre todo en la industria de las máquinas agrícolas, fueron decisivas para la expansión del sector en el oeste de los Estados Unidos de América en el siglo XIX, principalmente por medio de tecnologías de ahorro de mano de obra. Además de las innovaciones en la producción, las nuevas configuraciones productivas ya modificaban la producción agropecuaria del país en la década de 1950, como por ejemplo, la integración del sector del agro con los fabricantes de insumos y la agroindustria (Sunding y Zilberman, 2000). Como señala Alves (2010), las tecnologías de naturaleza químico-biológica, como los insumos que aumentan el rendimiento de la tierra, también incrementan el del trabajo. De ese modo, la productividad del trabajo, o producto por trabajador, depende tanto de la tecnología bioquímica como de la mecánica.

La mayor interacción de otros sectores con la industria agropecuaria estadounidense en la década de 1950 ya mostraba el declive de la producción del agro (dentro da porteira $)^{5}$ en el PIB. De acuerdo con Davis y

\footnotetext{
${ }^{5}$ El término dentro da porteira comprende todo lo relativo a la producción agrícola: siembra, manejo, cosecha, rendimientos, mantención de máquinas, almacenamiento de insumos, descarte de embalajes y mano de obra. Antes da porteira hace referencia a todo lo necesario para la producción agrícola, pero que no se encuentra en el establecimiento, y a aquello que el productor rural necesita comprar para producir: todos los insumos (máquinas, agentes químicos, fertilizantes, semillas y otros). En tanto que depois da porteira se refiere al almacenamiento y la distribución, incluida la logística.
}

Goldberg (1957), el término agronegocios o agroindustria (agribusiness) se definió como la suma de las operaciones en la compra de insumos, la producción y la distribución de productos agropecuarios, pues en ese período se verificó una mayor relación de la actividad agrícola con la cadena productiva antes e depois da porteira (la industria y el comercio que proporcionan insumos para la producción rural y la compra, el transporte, el procesamiento y la venta de los productos agropecuarios hasta el consumidor final, respectivamente).

Para Dimitri, Effland y Conklin (2005), la evolución tecnológica en la agricultura de los Estados Unidos de América comienza en el período posterior a la Segunda Guerra Mundial. Los fertilizantes químicos y pesticidas de bajo costo se utilizan desde 1945. Simultáneamente, se registran avances en el mejoramiento genético de plantas y animales y en la mecanización, que aumentan la rentabilidad de la actividad agropecuaria. A fines de los años sesenta se adopta la cosecha mecanizada de cultivos como la remolacha, el algodón y el tomate, mientras que en 1970 los tractores sustituyen a la fuerza animal. Desde 1900, las nuevas tecnologías y el desarrollo de infraestructura rural han fortalecido e incrementado los vínculos de los agricultores con los mercados de trabajo y capital y con muchos servicios ${ }^{6}$.

$\mathrm{Al}$ analizar las diferencias que se observan al comparar la evolución de la productividad de la agricultura en el Brasil y los Estados Unidos de América, es importante tener en cuenta que la fase inicial de comparación es diferente, pues el proceso de modernización de cada uno de ellos no tuvo lugar en un mismo momento. En este caso, siguiendo la tradición de la CEPAL, Rodríguez (1977) demuestra que muchas tecnologías se desarrollaban y adoptaban en los países más desarrollados (centro), y que algunas de ellas posteriormente se difundían en los países menos desarrollados (periferia).

\footnotetext{
${ }^{6}$ Vale la pena señalar los costos de transporte, que afectan de manera diferente al uso de los factores (mano de obra, tierra y tipo de tecnología) en los dos países. Véanse, por ejemplo, Chomitz y Gray (1996) y Cropper, Puri y Griffiths (2001). En cierta forma, se trata de un aspecto importante que la metodología adoptada no capta completamente.
} 


\section{III}

\section{Método de análisis: medición de la PTF}

El crecimiento de la PTF es la diferencia entre la tasa efectiva de crecimiento de la producción y la tasa de crecimiento relativa a los factores productivos, de no haber cambio tecnológico ni mejoramiento de la eficiencia de los productores. La tasa de crecimiento de la producción puede indicar dos situaciones distintas: una que se refiere al aumento de la cantidad física de insumos en el proceso productivo y otra a hacer más eficiente el uso de los factores en dicho proceso. Si los insumos se utilizan con mayor eficiencia, queda claro el crecimiento de la PTF.

De acuerdo con Sadoulet y De Janvry (1995), la PTF es la medida más común de progreso técnico, definido como una relación entre la producción (output) y un índice de todos los insumos (inputs). Para Pires y Garcia (2004), los cambios en la eficiencia de la asignación desempeñan un papel fundamental y aún más importante que el de la brecha tecnológica en la explicación de las diferencias de productividad entre países desarrollados y en desarrollo. Jorgenson (1996) y Christensen (1975) procuran detallar los conceptos y la construcción del índice que evalúa esta medida de productividad. En el cálculo de este indicador con respecto al Brasil se destacan Gasques y otros (2010).

Según esos autores, el índice de Tornqvist utilizado para calcular la PTF es una aproximación discreta del índice de Divisia (Chambers, 1998) y el más adecuado para el análisis de variables económicas, pues los datos están disponibles en forma discreta. En ese sentido:

$$
\begin{gathered}
\ln \left(\frac{P T F_{t}}{P T F_{(t-1)}}\right)=\frac{1}{2} \sum_{i=1}^{n}\left(S_{i t}+S_{i(t-1)}\right) \ln \left(\frac{Y_{i t}}{Y_{i(t-1)}}\right) \\
-\frac{1}{2} \sum_{j=1}^{m}\left(C_{j t}+C_{j(t-1)}\right) \ln \left(\frac{X_{j t}}{X_{j(t-1)}}\right)
\end{gathered}
$$

donde $Y_{i}$ y $X_{j}$ son, respectivamente, la cantidad producida y el volumen de los insumos, mientras que $S_{i}$ y $C_{j}$ se refieren a la proporción del producto $i$ en el valor total de la producción y a la porción del insumo $j$ en el costo total de los insumos.

En el lado izquierdo de la ecuación se define la variación de la PTF a lo largo de dos períodos sucesivos en el tiempo, mientras que en el lado derecho se incluyen dos términos. El primero es el logaritmo de la razón de las cantidades en dos períodos de tiempo, ponderado por la media de la proporción de cada producto en el valor total de la producción. El segundo es el logaritmo de la razón de las cantidades de insumos en el mismo período, también ponderado por la media de la proporción de cada insumo en el costo total.

En consecuencia, para calcular el índice de Tornqvist es necesario disponer de los precios y las cantidades de todos los productos e insumos utilizados. La variación de la PTF se calcula por función exponencial. Para obtener el índice de PTF de cada año, se selecciona un año base al que se le asigna el valor 100 y que luego se encadena con los años subsiguientes. Este proceso de encadenamiento puede estudiarse en Hoffmann (1980).

Para el cálculo de la PTF, de acuerdo con MAPA/ AGE (2011) y Gasques y otros (2014), se deben medir dos tipos de índices: i) producto agregado, y ii) insumo agregado. En el primer caso se utilizaron datos sobre cultivos permanentes y temporales y sobre producción y sacrificio de animales. El índice incluyó 66 productos correspondientes a cultivos (31 temporales y 35 permanentes) y 11 a la actividad pecuaria (8 productos de la cría de animales y 3 tipos de carne - bovina, porcina y de pollo-). Los datos del Instituto Brasileño de Geografía y Estadística (IBGE) sobre los valores y las cantidades se basan en las encuestas de Producción Agrícola Municipal (PAM) y de Producción Pecuaria Municipal (PPM). Las cifras referentes al peso de las carcasas se tomaron de la Encuesta Trimestral del Sacrificio de Animales del IBGE. Los precios corresponden a la encuesta de la Fundación Getulio Vargas.

En el caso del insumo agregado, el índice está formado por tres componentes, a saber: tierra (cultivos y pasturas), trabajo (personal ocupado) y capital (máquinas, pesticidas y fertilizantes). En el caso de la tierra, los datos relativos a las áreas cultivadas se tomaron de la encuesta PAM y los de área de pastoreo de los Censos Agropecuarios del IBGE. Las estadísticas de los años intercensales se calcularon por interpolación mediante las tasas de crecimiento. Los datos relativos al precio y al arrendamiento de la tierra provienen de la Fundación Getulio Vargas. En el caso del trabajo, los datos sobre la mano de obra (personas de 15 años de edad o más ocupadas en las actividades agrícolas) y los salarios se 
tomaron de la Encuesta Nacional de Hogares (PNAD) del IBGE. Con respecto al capital, las cantidades de máquinas agrícolas automotrices utilizadas se tomaron del anuario de la Asociación Nacional de Fabricantes de Vehículos Automotores (ANFAVEA), y los valores corresponden a la facturación de las empresas con unidades y piezas de repuesto vendidas internamente.

El período de depreciación considerado fue de 16 años. Así, cada 16 años se sustrae de la cantidad de unidades vendidas el número de unidades existentes hasta ese año, de modo que el resultado proporciona las existencias de máquinas del año en curso. Los datos de consumo de fertilizantes y pesticidas se obtuvieron mediante solicitudes. En el caso de los fertilizantes se pidió información a Potafos y a la Asociación Nacional para la Difusión de Fertilizantes (ANDA), mientras que en el de los pesticidas se contactó al Sindicato Nacional de la Industria de Productos para Defensa Agrícola (SINDIVEG).

Para calcular los índices no es necesario deflactar los valores de los productos y los insumos, pues se trabaja con las participaciones anuales. El período estudiado va de 1975 a 2010. En el caso de los Estados Unidos de América, las estadísticas se tomaron del Servicio de Investigación Económica del Departamento de Agricultura de los Estados Unidos. Cabe destacar que en el cálculo de las estadísticas brasileñas se utilizó la misma fórmula y metodología aplicadas por el Departamento de Agricultura de los Estados Unidos, justamente para proporcionar una buena base de comparación. Se debe tener en cuenta que el período estudiado es bastante largo y que tanto la estructura económica brasileña como la estadounidense cambiaron suficiente en ese tiempo. De cualquier manera, esto no invalida el estudio comparativo. Entre los trabajos en los que se realizan diversas comparaciones internacionales de la PTF, se sugiere la lectura del libro de Fuglie, Wang y Ball (2012). El crecimiento de la PTF brasileña fue uno de los más rápidos entre los diversos países analizados, como se aprecia también en el siguiente análisis comparado con la evolución estadounidense.

\section{IV}

\section{Comparación de la PTF entre el Brasil y los Estados Unidos de América}

\section{Evolución y panorama general}

Si bien la fase inicial de análisis de la PTF en un sector como el agropecuario o en la producción de un cultivo puede variar de un país a otro, el objetivo de este trabajo consiste en determinar si las brechas o diferencias entre el Brasil y los Estados Unidos de América han aumentado o disminuido con el paso del tiempo. La serie histórica analizada va de 1975 a 2013. Cabe destacar que las estructuras productivas brasileña y estadounidense son muy diferentes al comienzo y al final de ese período, y que la comparación de esas economías debe relativizarse en este contexto.

La productividad del sector agropecuario brasileño se ha examinado en muchos trabajos, entre ellos Gasques y Conceição (2000); Gasques, Villa Verde y Oliveira (2004); Gasques, Bastos y Bacchi (2008), y Gasques y otros (2010). Los principales análisis específicos de la evolución de la actividad agropecuaria estadounidense basados en cálculos de la PTF son los realizados por el Servicio de Investigación Económica del Departamento de Agricultura de los Estados Unidos. Sin embargo, no hay estudios comparativos de la evolución de los dos países para determinar si las brechas están aumentando o disminuyendo con el correr del tiempo. En este trabajo se optó por la comparación con los Estados Unidos de América porque este país presenta altos índices de productividad, mantiene un considerable volumen de producción agropecuaria y es considerado un líder mundial en la producción de muchos cultivos.

Los índices de PTF miden el agregado de producto por unidad de insumo agregado y ofrecen así una guía para observar la eficiencia de la producción agrícola (Thirtle y Bottomley, 1992). Son pocos los estudios en que se calcula la PTF por cultivos, debido a la dificultad de controlar los niveles específicos de insumo y producto. El hecho de que en una misma unidad de producción se compartan a menudo los insumos dificulta su contabilidad para un cultivo específico. Por ejemplo, la propia metodología del censo agropecuario brasileño determina la recolección de los datos de insumo, mano de obra y producto por establecimiento, y no por cultivo. 
Por ese motivo, el enfoque de la comparación es por sector agropecuario.

Antes de comparar la evolución de la PTF de la actividad agropecuaria brasileña y estadounidense, cabe examinar la evolución de ese indicador en otras regiones del mundo. En el gráfico 1 se muestra la PTF de los países de América Latina y el Caribe —incluido el Brasilen comparación con la de otras regiones y grupos de países, entre ellos los países de altos ingresos como los
Estados Unidos de América. Se observa que el grupo de ingresos elevados registró el mayor crecimiento medio de la PTF de 1961 a 2007, seguido por América Latina y el Caribe, cuyo crecimiento superó incluso la media mundial. Ludena (2010) destaca el buen desempeño económico de la región con respecto a las demás regiones en desarrollo: después de 1980 se incrementó la eficiencia y se redujo la brecha con los países de altos ingresos como los Estados Unidos de América.

Crecimiento anual de la PTF en la agricultura, promedio ponderado, 1961-2007 (En porcentajes)

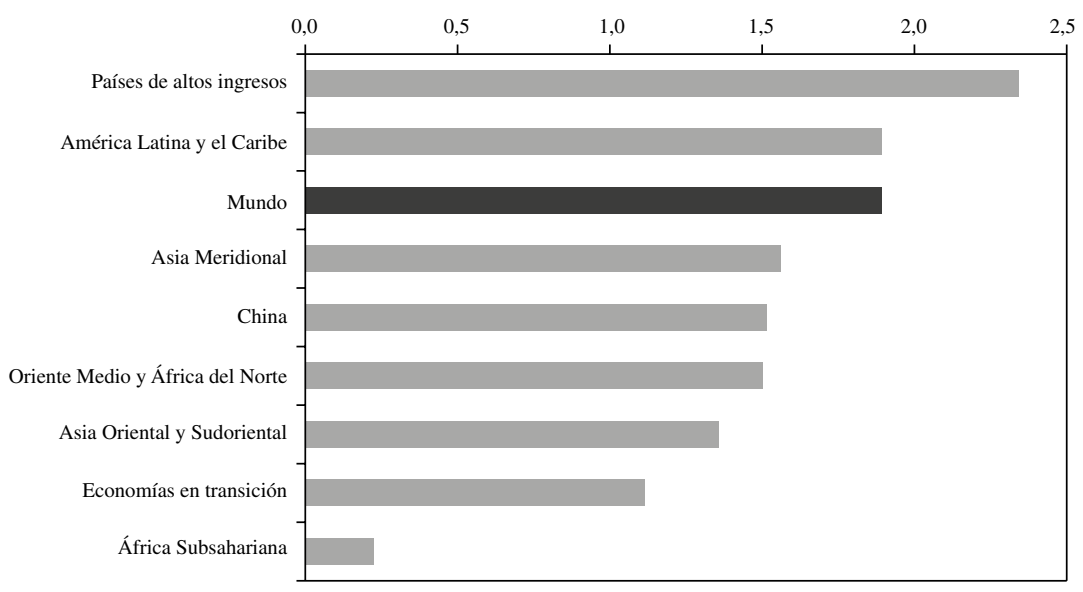

Fuente: C.E. Ludena, "Agricultural productivity growth, efficiency change and technical progress in Latin America and the Caribbean", IDB Working Paper Series, N ${ }^{\circ}$ 186, Washington, D.C., Banco Interamericano de Desarrollo (BID), 2010.

En el cuadro 1 se presentan los datos relativos al crecimiento de la producción agropecuaria y de la PTF agrícola entre 1961 y 2007, desglosados por década y respecto de todo el período examinado. Para ello, se toma como referencia a todos los países en desarrollo. Pese a que se dispone de los datos de América Latina y el Caribe, para un análisis más aproximado del Brasil se hace hincapié en el grupo denominado nordeste de América del Sur, donde el Brasil es el país principal, seguido por Guayana Francesa, Guyana y Suriname. Se puede apreciar la evolución de todos los países desarrollados y en particular de los Estados Unidos de América y el Canadá.

El grupo en que se incluye al Brasil registró un gran avance en el crecimiento de la producción y de la PTF agrícola, con excepción de algunos períodos - como el de 1961 a 1979- en que esta última no aumentó. En los demás períodos, el crecimiento productivo fue mayor que el de los países desarrollados y que el del grupo formado por los Estados Unidos de América y el
Canadá. Con respecto a la PTF, el mayor avance en el nordeste de América del Sur — que incluye al Brasiltuvo lugar a partir de la década de 1980 , período en que los porcentajes de crecimiento medio se distanciaron de los del resto de América Latina y el Caribe.

El análisis de la PTF solo en el Brasil revela un mayor incremento en los últimos años en comparación con las series más largas que abarcan las décadas de 1970 y 1980. En el cuadro 2, Gasques, Bastos y Bacchi (2008) indican los principales trabajos sobre el estudio de la PTF en el Brasil y los Estados Unidos de América.

La mayor evolución de la PTF en períodos más recientes confirma a los trabajos que describen el avance de la agricultura brasileña hacia nuevas fronteras agrícolas. La producción de granos en el Centro-Oeste, favorecida por el uso de insumos modernos y la mecanización, constituye un ejemplo. De acuerdo con Yokoyama e Igreja (1992), la participación de la soja de esa región en la agricultura brasileña pasó de apenas el $4 \%$ del área cultivada en 1975 al $28 \%$ en 1985. 
CUADRO 1

Producción agrícola y crecimiento de la productividad en regiones globales, por década, 1961-2007

(En porcentajes, promedio anual a lo largo del período)

\begin{tabular}{|c|c|c|c|c|c|c|c|c|c|c|c|c|}
\hline \multirow[b]{2}{*}{ Períodos } & \multicolumn{6}{|c|}{ Crecimiento de la producción agrícola } & \multicolumn{6}{|c|}{ Crecimiento de la PTF en la agricultura } \\
\hline & $\begin{array}{c}1961- \\
1969\end{array}$ & $\begin{array}{l}1970- \\
1979\end{array}$ & $\begin{array}{c}1980- \\
1989\end{array}$ & $\begin{array}{c}1990- \\
1999\end{array}$ & $\begin{array}{l}2000- \\
2007\end{array}$ & $\begin{array}{l}1961- \\
2007\end{array}$ & $\begin{array}{c}1961- \\
1969\end{array}$ & $\begin{array}{c}1970- \\
1979\end{array}$ & $\begin{array}{c}1980- \\
1989\end{array}$ & $\begin{array}{l}1990- \\
1999\end{array}$ & $\begin{array}{l}2000- \\
2007\end{array}$ & $\begin{array}{l}1961- \\
2007\end{array}$ \\
\hline Todos los países en desarrollo & 3,16 & 2,82 & 3,47 & 3,65 & 2,99 & 3,23 & 0,18 & 0,54 & 1,66 & 2,3 & 1,98 & 1,35 \\
\hline América Latina y el Caribe & 3,11 & 3,07 & 2,39 & 2,92 & 3,23 & 2,92 & 0,29 & 0,7 & 1,2 & 2,54 & 2,6 & 1,47 \\
\hline $\begin{array}{l}\text { Nordeste de América del Sur } \\
\text { (principalmente el Brasil) }\end{array}$ & 3,56 & 3,82 & 3,7 & 3,31 & 4,05 & 3,68 & $-0,52$ & $-0,76$ & 3,08 & 3,81 & 3,63 & 1,87 \\
\hline Todos los países desarrollados & 2,08 & 1,86 & 0,88 & 1,16 & 0,17 & 1,24 & 1,21 & 1,52 & 1,47 & 2,13 & 0,86 & 1,48 \\
\hline Estados Unidos de América y Canadá & 2,05 & 2,17 & 0,73 & 2,04 & 1,04 & 1,61 & 0,86 & 1,37 & 1,35 & 2,26 & 0,33 & 1,29 \\
\hline
\end{tabular}

Fuente: K.O. Fuglie, "Total factor productivity in the global agricultural economy: evidence from FAO data", The Shifting Patterns of Agricultural Production and Productivity Worldwide, J.M. Alston, B.A. Babcock y P.G. Pardey (eds.), Iowa, Universidad Estatal de Iowa, 2010.

Nota: PTF: Productividad total de los factores.

CUADRO 2

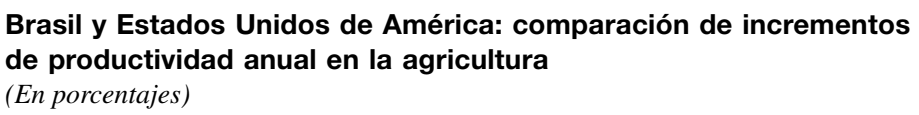

\begin{tabular}{lcc}
\hline Regiones & $\begin{array}{c}\text { Productividad total } \\
\text { de los factores (PTF) }\end{array}$ & 2,48 \\
\hline São Paulo (1995-2002) & 1,38 & (Vicente, 2003) \\
Estados Unidos de América (1999-2002) & 2,51 & (Ball, 2006) \\
Brasil (1975-2005) & 3,87 & (Gasques, Bastos y Bacchi, 2007) \\
Brasil (2000-2005) & (Gasques, Bastos y Bacchi, 2007) \\
\hline
\end{tabular}

Fuente: J.G. Gasques, E.T. Bastos y M.R.P. Bacchi, "Produtividade e fontes de crescimento da agricultura brasileira", Políticas de incentivo à inovação tecnológica, J.A. de Negri y L.C. Kubota (eds.), Brasilia, Instituto de Investigación Económica Aplicada (IPEA), 2008.

En el cuadro 3 se muestran los índices de crecimiento en períodos seleccionados para comparar la evolución de otros factores, como la productividad de la tierra y de la mano de obra. Cabe recordar que la productividad del trabajo, o producto por trabajador, depende tanto de la tecnología bioquímica como de la mecánica.
Vieira Filho y Silveira (2011) subrayan la importancia del conocimiento, que puede utilizarse para mejorar las técnicas de producción. Ese conocimiento puede ser tácito o basarse en los trabajos de investigación de instituciones como la Empresa Brasileña de Investigación Agropecuaria (EMBRAPA).

CUADRO 3

Brasil: tasas anuales de crecimiento, 1970-2006 y 1995-2006

(En porcentajes)

\begin{tabular}{lcc}
\hline Indicadores & $1970-2006$ & $1995-2006$ \\
\hline Índice de producto & 3,483 & 3,138 \\
Índice de insumos & 1,189 & 0,991 \\
PTF & 2,267 & 2,126 \\
Productividad de la tierra & 3,316 & 3,158 \\
Productividad de la mano de obra & 3,528 & 3,409 \\
\hline
\end{tabular}

Fuente: J.G. Gasques, "Produtividade total dos fatores e transformações da agricultura brasileira: análise dos dados dos censos agropecuários", A agricultura brasileira: desempenho, desafios e perspectivas, J.G. Gasques, J.E.R. Vieira Filho y Z. Navarro (orgs.), Brasilia, Instituto de Investigación Económica Aplicada (IPEA), 2010.

Nota: PTF: Productividad total de los factores. 
Al realizar un corte en la evolución de los índices entre 1970-2006 y 1995-2006, se observa que el crecimiento del producto se debe principalmente al crecimiento de la PTF y que la productividad de la mano de obra fue mayor que la de la tierra en ambos períodos. Sin embargo, como indican Gasques y otros (2010), la productividad de la mano de obra se refiere a una productividad bruta, cuyo componente principal es la productividad de la tierra. Así, el empleo de una tecnología de ahorro de tierra, como un fertilizante, puede coadyuvar a una mayor productividad del trabajo en la medida en que operaciones como la cosecha pueden realizarse con menos personas y máquinas.

Como demuestran Sunding y Zilberman (2000), el proceso de modernización estadounidense mediante la adopción de tecnologías de ahorro de tierra y mano de obra comenzó en la década de 1950, mucho antes que en el Brasil, donde esto ocurrió en las décadas siguientes.

En el gráfico 2 se muestra la evolución de los índices de insumos, producto y PTF en los Estados Unidos de América de 1948 a 2009. El índice de insumos en la agricultura varía poco, de manera que el crecimiento de la PTF se debe al aumento del nivel de productos. De acuerdo con Gasques y otros (2010), algunos factores, como la investigación agropecuaria, tienen gran importancia en el incremento de la productividad. La mejor asignación de los recursos —incluidas las tecnologías más modernas que reducen las pérdidas en el uso de los insumos (fertilizantes con menor lixiviación, fijación biológica de nutrientes, variedades mejoradas, conocimiento acumulado y aprendizaje, entre otros) permite aumentar la producción (output) con menor cantidad de insumos (input).

Existen muchos factores que posibilitan una mayor eficiencia en la gestión de los recursos en la actividad agropecuaria y en el objetivo de optimizar su uso. La investigación agropecuaria por parte de empresas públicas y privadas es importante para la creación de innovaciones tecnológicas, generalmente por medio de nuevos procesos de producción. Como observa Vieira Filho (2009), la adopción de la siembra directa sobre rastrojo, utilizada en gran escala en el Brasil para el cultivo de cereales, es fruto de esa interacción en que el aprendizaje adquiere gran importancia. El cambio de una tecnología, como la siembra directa o un fertilizante con mayor nivel de absorción por las plantas, permite producir más (mayor productividad) con menor cantidad de insumos. Coelli y Prasada Rao (2005) señalan que las diferencias en el acceso a algunos factores (fertilizantes, tractores, animales e irrigación, entre otros) pueden promover diferencias de productividad entre países. Por otra parte, Ruttan (2002) indica algunos problemas enfrentados por los agricultores de todo el mundo que alteran la productividad de la agricultura, como la degradación y la pérdida de suelos, las inundaciones y la salinidad, la coevolución de plagas, patógenos y huéspedes, y el cambio climático.

GRÁFICO 2

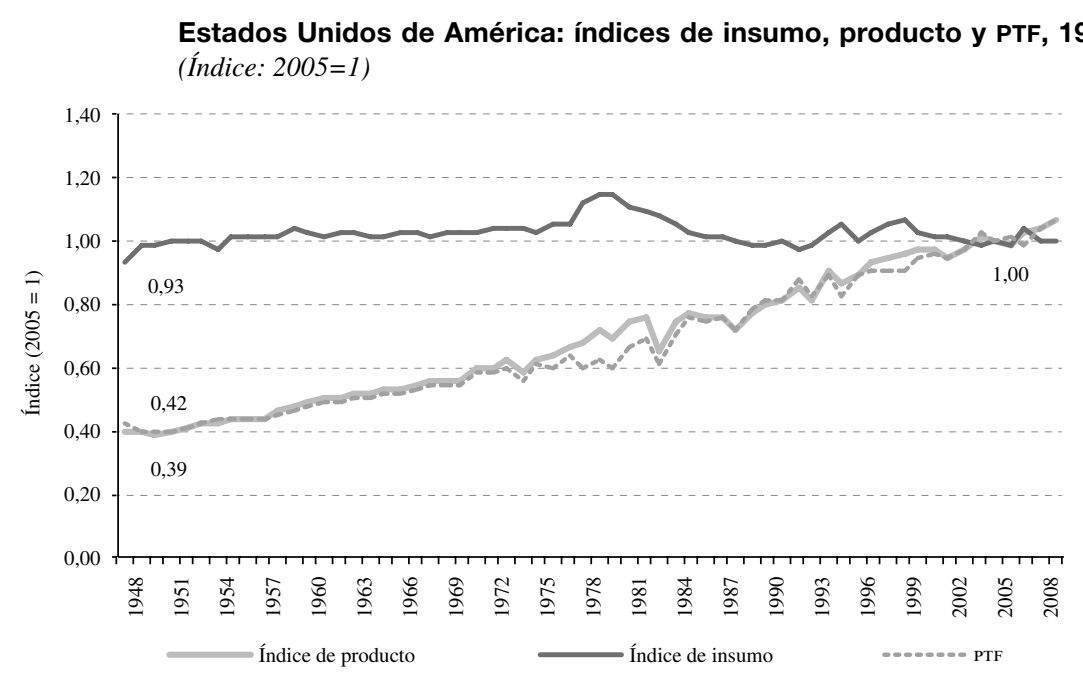

Fuente: Departamento de Agricultura de los Estados Unidos, “Agricultural Productivity in the U.S.", Servicio de Investigación Económica, 2012 [en línea] http://www.ers.usda.gov/Data/AgProductivity.

Nota: PTF: Productividad total de los factores. 
En el cuadro 4 se presentan las principales fuentes de crecimiento de la agricultura de los Estados Unidos de América de 1948 a 2009. Al examinar la evolución del trabajo se observa que este indicador fue negativo en todos los períodos, es decir, que se pasó a utilizar menos mano de obra.

El índice de producto fue positivo en toda la serie, y en algunos períodos — como el de 1966-1979— registró un crecimiento mayor. En el caso de la tierra no hubo grandes variaciones con el correr del tiempo. El capital aumentó más entre 1948 y 1953, un período en que, de acuerdo con Sunding y Zilberman (2000), los agricultores estadounidenses comenzaron a utilizar más tecnología (máquinas agrícolas y fertilizantes) y eso se tradujo en una mayor necesidad de capital. Excepto entre 1979 y 1990 y en el período más reciente (2007-2009), la fuente denominada materiales o entrada de materiales, que incluye el uso de energía, fertilizantes y químicos, presenta valores crecientes. Esto demuestra que en la agricultura estadounidense se utilizan cada vez más productos provenientes de otros sectores, como por ejemplo la industria.

La agricultura brasileña ha crecido a tasas similares (o incluso superiores) a las de los Estados Unidos de América, de modo que la brecha entre los dos países se ha reducido. Sin embargo, la composición de los factores no es la misma, pues se encuentran en distintas etapas de evolución de la agricultura. Ludena (2010) subraya que se debe tener cuidado en la interpretación, pues los niveles de desarrollo de los países son diferentes. Por ejemplo, en el estudio de Alauddin, Headey y Prasada Rao (2005) se revela que la PTF del Brasil en 1970 era la mitad de la PTF de los Estados Unidos de América. Los factores climáticos pueden influir en la producción y determinar una reducción del producto, que a su vez provoca una disminución de la PTF. Los cambios en las expectativas de precios en los mercados pueden incentivar o desestimular una actividad, e incluso conducir a su reemplazo por otra menos eficiente desde el punto de vista de la producción.

CUADRO 4

\begin{abstract}
Estados Unidos de América: fuentes de crecimiento en el sector agrícola y tasas promedio de crecimiento anual, 1948-2009

(En porcentajes)
\end{abstract}

\begin{tabular}{|c|c|c|c|c|c|c|c|c|c|c|c|c|c|}
\hline & $\begin{array}{c}1948- \\
2009\end{array}$ & $\begin{array}{c}1948- \\
1953\end{array}$ & $\begin{array}{c}1953- \\
1957\end{array}$ & $\begin{array}{c}1957- \\
1960\end{array}$ & $\begin{array}{c}1960- \\
1966\end{array}$ & $\begin{array}{c}1966- \\
1969\end{array}$ & $\begin{array}{c}1969- \\
1973\end{array}$ & $\begin{array}{c}1973- \\
1979\end{array}$ & $\begin{array}{c}1979- \\
1981\end{array}$ & $\begin{array}{c}1981- \\
1990\end{array}$ & $\begin{array}{l}1990- \\
2000\end{array}$ & $\begin{array}{l}2000- \\
2007\end{array}$ & $\begin{array}{l}2007- \\
2009\end{array}$ \\
\hline Pro & 1,63 & 1,18 & 0,96 & 4,03 & 1,21 & 2,24 & 2,65 & 2,26 & 1,54 & 0,96 & 1,84 & 0,77 & 1,88 \\
\hline Insumo & 0,11 & 1,34 & 0,28 & 0,50 & 0,05 & $-0,08$ & 0,46 & 1,64 & $-1,85$ & $-1,22$ & 0,31 & 0,14 & $-1,80$ \\
\hline
\end{tabular}

Fuentes

\begin{tabular}{lrrrrrrrrrrrrr}
\hline Trabajo & $-0,52$ & $-0,81$ & $-1,08$ & $-0,83$ & $-0,81$ & $-0,61$ & $-0,38$ & $-0,19$ & $-0,22$ & $-0,43$ & $-0,34$ & $-0,35$ & $-0,64$ \\
Capital & 0,02 & 0,54 & 0,15 & 0,03 & 0,08 & 0,32 & 0,14 & 0,32 & 0,23 & $-0,61$ & $-0,21$ & 0,05 & 0,35 \\
Tierra & $-0,08$ & 0,02 & $-0,17$ & $-0,16$ & $-0,07$ & $-0,22$ & $-0,29$ & 0,00 & $-0,12$ & $-0,09$ & 0,00 & $-0,08$ & $-0,12$ \\
Materiales & 0,69 & 1,58 & 1,38 & 1,45 & 0,85 & 0,43 & 0,99 & 1,50 & $-1,74$ & $-0,09$ & 0,87 & 0,52 & $-1,39$ \\
PTF & 1,52 & $-0,16$ & 0,68 & 3,53 & 1,16 & 2,32 & 2,19 & 0,62 & 3,39 & 2,19 & 1,53 & 0,63 & 3,68 \\
\hline
\end{tabular}

Fuente: Departamento de Agricultura de los Estados Unidos, “Agricultural Productivity in the U.S.”, Servicio de Investigación Económica, 2012 [en línea] http://www.ers.usda.gov/Data/AgProductivity.

Nota: PTF: Productividad total de los factores.

\section{Cuadro comparativo: reducción de la brecha productiva}

Para medir la brecha productiva internacional es necesario comparar la productividad nacional con la referencia internacional, que en este caso es la producción agropecuaria estadounidense, tanto por la tecnología aplicada como por los elevados índices de productividad. En el gráfico 3 se compara la PTF del Brasil con la de los Estados Unidos de América. Como se puede apreciar, la PTF brasileña registró un mayor crecimiento en comparación con la estadounidense después de 1975, de modo que la brecha de productividad entre los dos países se redujo. 
GRÁFICO 3

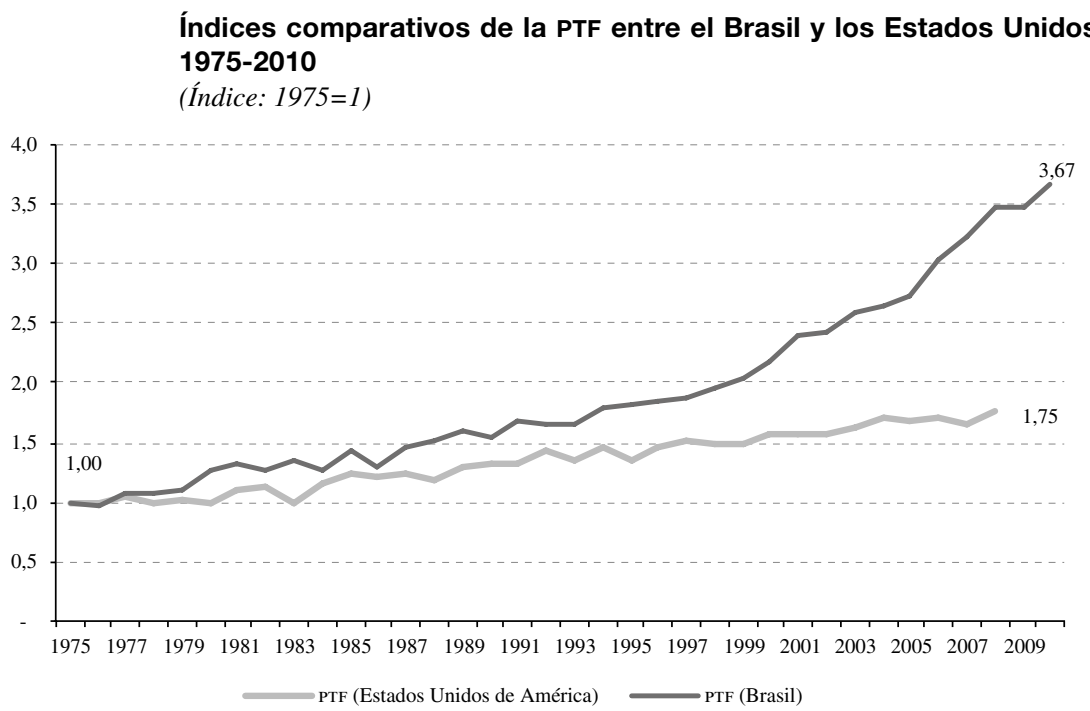

Fuente: Departamento de Agricultura de los Estados Unidos, Total Factor Productivity, Washington, D.C., Servicio de Investigación Económica, 2008; y Ministerio de Agricultura, Ganadería y Abastecimiento/Asesoría de Gestión Estratégica (MAPA/AGE), Produtividade total dos fatores, Brasilia, 2011.

Nota: PTF: Productividad total de los factores.

Se observa que el valor de los índices al final del período examinado fue de 3,67 en el Brasil (año 2010) y de 1,75 en los Estados Unidos de América (año 2008), de manera que mientras en el primer caso hubo un crecimiento del $267 \%$, en el segundo caso fue del $75 \%$. Los cambios que condujeron a un marcado aumento de la productividad en la agricultura estadounidense tuvieron lugar entre 1950 y 1970. En el caso del Brasil, el proceso de modernización comenzó con la importación de máquinas agrícolas en los años cincuenta, pero fue solo a partir de la década de 1970 que la productividad se incrementó en forma significativa, sobre todo con la planificación de la investigación agropecuaria. Además, la expansión de la frontera agrícola en el Brasil ocurrió a partir de 1970, un panorama probablemente distinto con respecto a los Estados Unidos de América.

En el estudio de la Organización para la Cooperación y el Desarrollo Económicos (OCDE, 2011) se presentan algunas estadísticas de las tasas de crecimiento de la PTF en diversas regiones del mundo. Mientras esa tasa tiende a disminuir en las economías desarrolladas, donde pasa del 1,48\% anual en el período 1961-2007 al 0,86\% anual en el período 2000-2007, tiende por el contrario a aumentar en los países en desarrollo, pasando del $1,35 \%$ anual en el período 1961-2007 al 1,98\% anual en el período 2000-2007.
En el gráfico 4 se comparan la PTF y los índices de insumo y producto del Brasil con los de los Estados Unidos de América. Esto permite determinar si el crecimiento de la producción obedece a la intensificación de los insumos o a la incorporación de tecnología que mejora la eficiencia productiva.

En el período analizado, el índice de insumos de la producción agropecuaria permaneció casi estable (con un crecimiento de apenas un 5\%), mientras que el índice de producto aumentó en $284 \%$. La mayor parte del incremento de la producción se debe a los cambios tecnológicos y significa que se produce más con menos recursos. Según los datos presentados, la media anual de crecimiento de la PTF en el Brasil entre 1975 y 2010 fue de 3,6\%, porcentaje superior al observado en los Estados Unidos de América $(1,9 \%)^{7}$. El índice de insumos se redujo un 7\% en ese país, al pasar de 1,00 en 1975 a 0,93 en 2008. No obstante, se observa que el producto y la PTF aumentaron en $62 \%$ y $75 \%$, respectivamente, de manera que también se logra producir más con menos insumos.

\footnotetext{
${ }^{7}$ Véanse comparaciones en Ball (2006) y Gasques y otros (2012).
} 


\section{Estados Unidos de América}

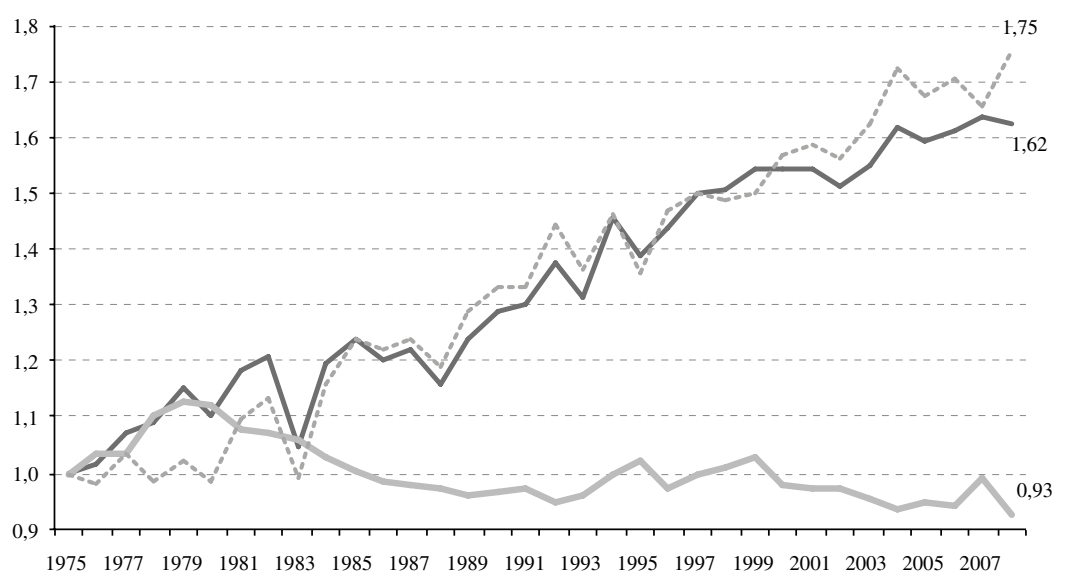

\section{Brasil}

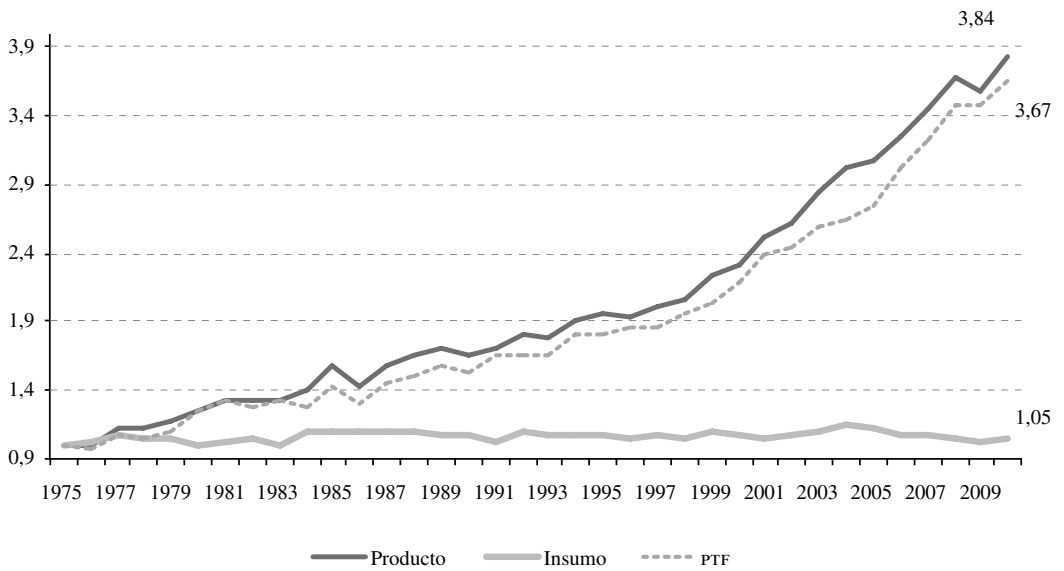

Fuente: Departamento de Agricultura de los Estados Unidos, Total Factor Productivity, Washington, D.C., Servicio de Investigación Económica, 2008; y Ministerio de Agricultura, Ganadería y Abastecimiento/Asesoría de Gestión Estratégica (MAPA/AGE), Produtividade total dos fatores, Brasilia, 2011.

Nota: PTF: Productividad total de los factores.

En el gráfico 5 se compara la utilización de trabajo, tierra y capital en la producción de los Estados Unidos de América y del Brasil. Si bien se aprecia un crecimiento del uso de capital en la economía estadounidense entre 1975 y 1981, al analizar el período de 1975 a 2010 se constata una contracción en el uso de los tres factores considerados. En el caso del capital, la reducción tiene lugar a partir de 1981. Ball y otros (2001) indican que el uso de capital fue importante en la agricultura estadounidense entre 1973 y 1981, pero su importancia disminuyó entre 1982 y 1993 porque la inversión neta en capital fijo es negativa en ese último período. Esto sugiere la obsolescencia del acervo de capital, tal vez debido al alto precio de la energía, que puede inhibir el aumento de la productividad.

La contracción del capital en la economía estadounidense es otro indicio de los avances tecnológicos, pues, como se vio anteriormente, el índice de producto tendió a aumentar en el mismo período. En el Brasil se observan una acentuada expansión del factor capital en todo el período, la incorporación de nuevas fronteras agrícolas hasta fines de la década de 1980, una relativa 
Estados Unidos de América

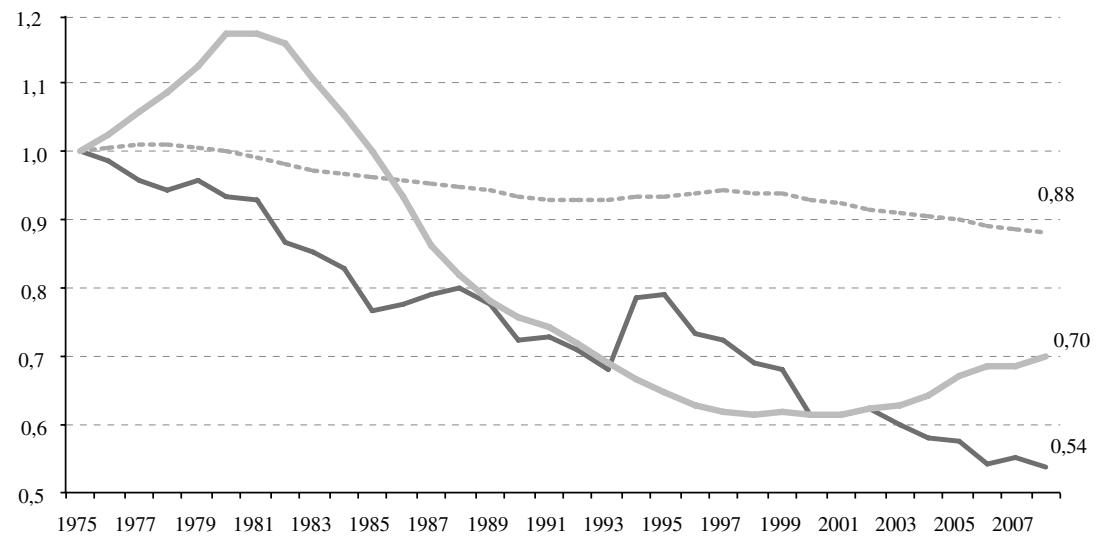

Brasil

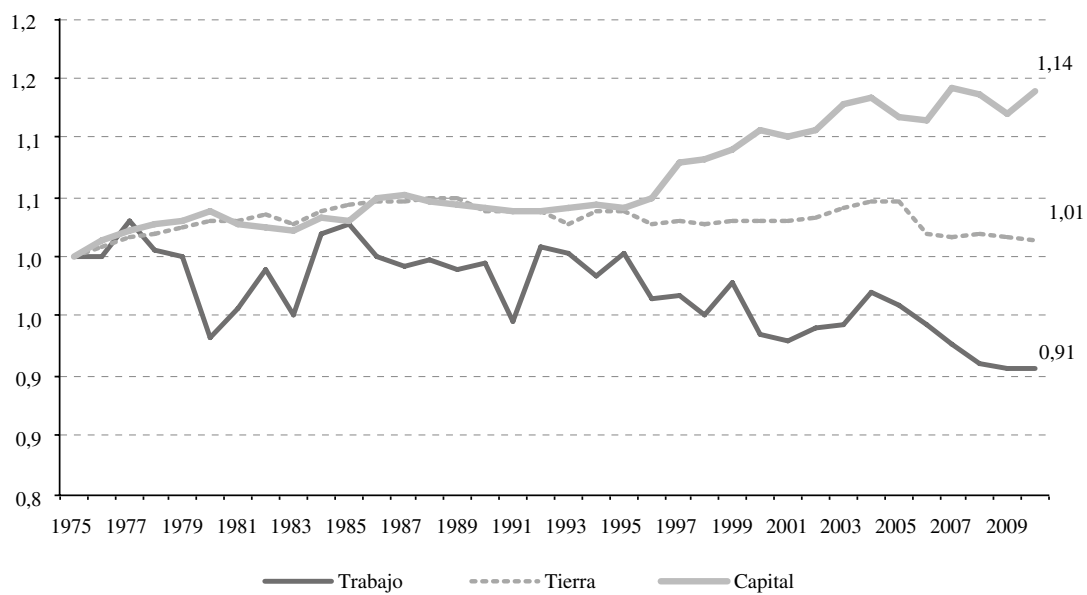

Fuente: Departamento de Agricultura de los Estados Unidos, Total Factor Productivity, Washington, D.C., Servicio de Investigación Económica, 2008; y Ministerio de Agricultura, Ganadería y Abastecimiento/Asesoría de Gestión Estratégica (MAPA/AGE), Produtividade total dos fatores, Brasilia, 2011.

estabilización del uso de tierras a partir de la década de 1990 y una considerable reducción del factor trabajo a partir de 1990. El crecimiento del capital y la disminución del trabajo se observan sobre todo en esos años, que coinciden con la apertura comercial y mayores inversiones privadas en la agricultura.

El aumento del índice de capital se asocia a la creación de programas de financiamiento e inversión en la agricultura brasileña a partir de la segunda mitad de la década de 1990. Se destaca en primer lugar el Programa Nacional de Agricultura Familiar (PRONAF), seguido por el Programa de modernización de la flota de tractores agrícolas e implementos asociados y cosechadoras (MODERFROTA). Esos programas fueron fundamentales para la modernización y el avance tecnológico del capital en la agricultura brasileña y condujeron a un aumento del $14 \%$ en el período estudiado.

De acuerdo con Gasques y otros (2010, pág. 35), el incremento de la productividad de la tierra en el caso brasileño se debe al acrecentamiento de los gastos en investigación, especialmente de la Embrapa, y a la incorporación de nuevas áreas con mayor productividad 
en parte del período de referencia de más de 30 años. Los autores señalan que el aumento de la productividad de la tierra obedece también a las innovaciones introducidas en los procesos de producción, entre ellas la investigación agropecuaria, el sistema de siembra directa, la inoculación con bacterias, el manejo integrado de plagas y la creación de variedades y especies con plasticidad suficiente para adaptarse a las diferentes condiciones climáticas y ambientales. Gasques y otros (2013) muestran que el desplazamiento de la producción de cultivos y de la actividad pecuaria a nuevas regiones del Norte, Centro Oeste y Centro Nordeste, así como la calidad de los insumos utilizados en la actividad agropecuaria, entre otros factores, también contribuyeron a acrecentar la productividad.

La participación de las universidades en la investigación para la agricultura también es muy importante para el Brasil. Teixeira, Clemente y Braga (2013) señalan la relevancia de los programas de posgrado en los trabajos de investigación en áreas como el mejoramiento de plantas y animales, suelos y fertilidad, mecanización, manejo de plantas y animales y en la creación de productos que permitieron el desarrollo de la agroindustria en el país.

Con respecto a la convergencia de la productividad agropecuaria del Brasil con la de los Estados Unidos de América, se observa que la brecha entre los dos países ha disminuido. La agricultura brasileña ha crecido de manera muy intensa, volviéndose más productiva, menos intensiva en trabajo y con una cantidad estable de tierras utilizadas.

\section{Desigualdad productiva de la agricultura brasileña $^{8}$}

Al comparar el sector agropecuario brasileño con el estadounidense se advierte una convergencia de la PTF, que reduce las distancias productivas entre los dos países. Sin embargo, esa convergencia no abarca la totalidad de la producción brasileña, debido al elevado grado de heterogeneidad estructural que existe en el segmento productivo.

Si bien los resultados de la PTF son convergentes, la heterogeneidad y complejidad regional del Brasil

\footnotetext{
${ }^{8}$ El objetivo de esta subsección consiste en mostrar brevemente que los incrementos de productividad mencionados se incorporan de forma diferenciada en el Brasil, sobre todo en virtud de la elevada concentración productiva existente, que se refleja en términos regionales y sectoriales. No corresponde hacer aquí una investigación exhaustiva de estos temas. Véanse estudios más profundos y detallados del tema en Fornazier y Vieira Filho (2012); Vieira Filho, Santos y Fornazier (2013).
}

determinan que no todo el sector agropecuario esté actualizado en materia tecnológica, de manera que solo una parte del sector productivo se apropia de los efectos de la modernización (Vieira Filho, 2012). Como señalan Poudel, Paudel y Zilberman (2011), es posible que haya convergencia entre regiones dentro de un país, pero no en el territorio nacional en su conjunto. Esto significa que, a pesar de la convergencia entre algunas regiones, los índices de crecimiento pueden variar mucho dentro de un país, manteniéndose las heterogeneidades regionales, que también pueden referirse a cultivos y actividades.

Con respecto a la desigualdad de ingresos, Fornazier y Vieira Filho (2012) y Vieira Filho, Santos y Fornazier (2013) señalan que la heterogeneidad productiva en la agricultura brasileña es muy pronunciada. $\mathrm{Al}$ analizar los resultados relativos a la agricultura familiar, Vieira Filho (2013) llega a una conclusión similar con respecto a la heterogeneidad productiva.

De acuerdo con el cuadro 5, los establecimientos agropecuarios pueden subdividirse según cuatro niveles de ingresos: extrema pobreza, bajos, medianos y altos ingresos. Los resultados muestran que los grupos de medianos y altos ingresos, que constituyen menos del $10 \%$ de los establecimientos agropecuarios, produjeron el $85 \%$ del valor bruto de producción (VBP), en tanto que el grupo en condiciones de extrema pobreza, o sea, el $63 \%$ de los establecimientos, participó con menos del $4 \%$ del vBP. El cálculo del coeficiente de Gini productivo presentó un indicador con elevada desigualdad, pues su valor fue muy cercano a 1 .

El grupo en condiciones de extrema pobreza, que abarca a cerca de 3,2 millones de establecimientos, está al margen de la producción agropecuaria y resulta excluido de todos los sectores de actividad económica, pues carece de estructuras básicas de organización productiva (micro y macroeconómicas).

El grupo de bajos ingresos ( 960.000 establecimientos agropecuarios) necesita asistencia del gobierno mediante políticas de fomento y dinamización de la producción en pequeña escala, normalmente de base familiar. Se trata de establecimientos con escasa capacidad de absorción de conocimiento externo y bajo contenido tecnológico, que presentan deficiencias en los ámbitos gerencial y microeconómico. Es preciso mejorar el acceso de estos productores al crédito y fomentar el uso de nuevas tecnologías. Cabe al gobierno mejorar el acceso a la asistencia técnica y desarrollar investigaciones de dominio público cuando el mercado no las ofrece.

Por último, la riqueza agrícola se concentra en los grupos de medianos y altos ingresos. Para esos grupos, la capacidad de absorción tecnológica constituye un problema 
CUADRO 5

Brasil: estratificación de ingresos de los establecimientos por producción, 2006

\begin{tabular}{|c|c|c|c|c|c|c|}
\hline Grupos & $\begin{array}{c}\text { Salario mínimo } \\
\text { mensual equivalente }\end{array}$ & $\begin{array}{l}\text { Número de } \\
\text { establecimientos } \\
(\text { en miles })\end{array}$ & Porcentaje & $\begin{array}{c}\text { Valor bruto de la } \\
\text { producción (VBP) anual } \\
\text { (en miles de millones) }\end{array}$ & Porcentaje & $\begin{array}{c}\text { Coeficiente } \\
\text { de Gini }\end{array}$ \\
\hline Extrema pobreza & $(0$ a 2$)$ & 3242 & 69,6 & 6,5 & 3,9 & \\
\hline Bajos ingresos & $(2$ a 10$)$ & 960 & 20,9 & 18,5 & 11,1 & \\
\hline Medianos ingresos & $(10$ a 200$)$ & 416 & 9 & 59,9 & 35,9 & 0,89 \\
\hline \multirow[t]{2}{*}{ Altos ingresos } & $>200$ & 23 & 0,5 & 81,7 & 49 & \\
\hline & Total & 4641 & 100 & 166,7 & 100 & \\
\hline
\end{tabular}

Fuente: Instituto Brasileño de Geografía y Estadística (IBGE), Censo Agropecuário 2006, Río de Janeiro, 2006.

a Salario mínimo equivalente $=$ valor bruto de la producción (VBP) mensual/salario mínimo mensual.

secundario, pero el ambiente macroeconómico favorable al crecimiento de las ventas se vuelve fundamental. Las políticas públicas deberían orientarse por temas que van más allá de los aspectos microeconómicos, centrándose en el estímulo de la competitividad, la promoción de las exportaciones, el seguro agrícola y el mejoramiento de la distribución logística de los productos.

La diferencia de productividad de un cultivo en determinado estado o región también refleja diferencias en la PTF: en algunas regiones, los productores se dedican a los cultivos más propicios según las condiciones del clima y los suelos, y realizan una mejor asignación de recursos, como los insumos, lo que supone una variación de la PTF. Gasques y otros (2010) indican que la PTF del sector agropecuario brasileño registró una media de crecimiento anual del 2,27\% en el período 1970-2006, mientras que en algunos estados, como el de Amazonas, el crecimiento fue negativo (-0,902\% anual). En otros, entre ellos Mato Grosso, el crecimiento fue positivo (4,67\% anual) en el mismo período. Estos resultados demuestran que, si bien las brechas entre países pueden disminuir, las diferencias intrarregionales brasileñas pueden persistir. En los Estados Unidos de América también hay diferencias de productividad entre los estados, como puede apreciarse en las estadísticas (Departamento de Agricultura de los Estados Unidos, 2012).

Las políticas públicas, así como las inversiones en investigación agropecuaria, asistencia técnica y extensión rural, pueden contribuir a que los agricultores hagan un mejor uso de los recursos o, en otras palabras, obtengan una mayor productividad utilizando menos recursos. Además de aumentar la producción, la reducción del uso de insumos en el sector agropecuario puede tener efectos deseables de naturaleza ambiental, laboral y de salud pública, en la medida en que mejora la optimización y disminuye la presión sobre los recursos naturales (Blandford, 2012).

De esa manera, es posible diseñar políticas públicas para regiones específicas y determinar, por ejemplo mediante la zonificación agroclimática, los lugares donde se puede obtener mayor productividad. No obstante, las políticas relacionadas con ese objetivo deben tener en cuenta distintos aspectos de inserción social en el mercado de trabajo y de preservación ambiental.

\section{V}

\section{Consideraciones finales}

De acuerdo con la CEPAL (2010), existen muchas diferencias en el nivel de desarrollo de los países e incluso dentro de un mismo país o sector, las que constituyen la heterogeneidad estructural. No obstante, las brechas pueden disminuir con el tiempo y con ellas también dicha heterogeneidad.

Para medir la diferencia entre países o sectores, en este estudio se comparó la evolución de la PTF en el sector agropecuario del Brasil y de los Estados Unidos de América y se observó que la brecha productiva tiende a disminuir. Sin embargo, la modernización de la actividad agropecuaria y su vinculación con otros sectores de la economía (como el de insumos) tienen lugar en distintos períodos en los dos países. En consecuencia, dicha reducción no significa necesariamente una aproximación de los índices de productividad de los dos países, pues 
entran en juego otros factores - como el nivel de conocimiento acumulado en una actividad- que no solo pueden promover diferencias de crecimiento, sino también ratificar la distancia existente.

Los países aquí analizados registraron un aumento de la PTF en una serie más larga, de manera que lograron una mayor producción con menor cantidad de insumos. Para ello se utilizaron tecnologías que permiten el ahorro de tierra, como los fertilizantes y otros productos agroquímicos, y aquellos que ahorran en trabajo, como son las maquinarias y los implementos agrícolas.

Con el uso más intensivo de algunos insumos y la sustitución de tecnologías en favor de uno u otro recurso, la productividad entre actividades puede disminuir entre países. No obstante, las diferencias climáticas o la capacidad de adopción de determinada tecnología, entre otros factores, dificultan las ganancias de productividad para todos. Si bien la brecha productiva entre el Brasil y los Estados Unidos de América ha disminuido, se observan muchas diferencias dentro de estos países. En otras palabras, la heterogeneidad estructural no solo existe entre los países desarrollados y en desarrollo, sino también dentro de un mismo país o actividad.

Las políticas públicas (como las inversiones en investigación y desarrollo (I+D), asistencia técnica y extensión rural) pueden ayudar a los productores a mejorar su eficiencia en la producción de alimentos y la crianza de animales para obtener un rendimiento con menos insumos y recursos. Además de aumentar la PTF, también contribuyen a la optimización de dichos medios y a la disminución de los problemas ambientales.
Dado el grado de heterogeneidad y complejidad regional, no todo el sector agropecuario brasileño está actualizado en materia tecnológica, de manera que solo una parte de ese sector se apropia de los efectos de la modernización. Al comparar el sector agropecuario brasileño con el estadounidense, se observa una convergencia de la PTF (o disminución de la brecha productiva), que aminora las distancias productivas entre los dos países. Sin embargo, esa convergencia no abarca la totalidad de la producción brasileña debido al elevado grado de heterogeneidad estructural existente y a la concentración productiva entre los agentes. A lo largo de las últimas décadas, la agricultura brasileña creció aceleradamente, volviéndose más productiva, menos intensiva en trabajo y con una cantidad estable de tierras utilizadas. Sin embargo, todavía existen enormes desafíos relacionados con la promoción del desarrollo inclusivo de los agentes del sector.

La heterogeneidad estructural impide la inserción productiva de los segmentos más rezagados en los mercados más dinámicos. Desde el punto de vista de la elaboración de políticas públicas, el estudio indica que hay una falla en el desarrollo inclusivo, que persistirá en la medida en que se mantengan las disparidades estructurales en la producción agrícola brasileña. Existen problemas micro y macroeconómicos que se diferencian de acuerdo con las especificidades de cada grupo productivo, y que también deben tenerse en cuenta en la elaboración de políticas de Estado que puedan corregir las fallas y minimizar los problemas de orden estructural.

\section{Bibliografía}

Alauddin, M., D. Headey y D.S. Prasada Rao (2005), "Explaining agricultural productivity levels and growth: an international perspective", Discussion Paper, $\mathrm{N}^{\circ}$ 2, Brisbane, Universidad de Queensland.

Alves, E.O. (2010), "O que significam as medidas de produtividade da agricultura?", Revista de Economia e Agronegócio, vol. 8, $\mathrm{N}^{\circ} 3$.

Ball, V.E. (2006), "Productivity and output growth in U.S. agriculture", Agricultural Resources and Environmental Indicators, K. Wiebe y N. Gollehon (eds.), Washington, D.C., Departamento de Agricultura de los Estados Unidos/Servicio de Investigación Económica.

Ball, V.E. y otros (2001), "Levels of farm sector productivity: an international comparison", Journal of Productivity Analysis, vol. 15, $\mathrm{N}^{\circ} 1$, Springer.

Blandford, D. (2012), "The Contribution of Agriculture to Green Growth" [en línea] http://www.oecd.org/dataoecd/ 25/41/48258861.pdf.

Buainain, A.M. y otros (orgs.) (2014), O mundo rural do Brasil no século 21: a formação de um novo padrão agrário e agrícola, Brasilia, Embrapa.

CEPAL (Comisión Económica para América Latina y el Caribe) (2010), "Heterogeneidad estructural y brechas de productividad: de la fragmentación a la convergencia”, La hora de la igualdad: brechas por cerrar, caminos por abrir (LC/G.2432(SES.33/3)), Santiago.

Chambers, R.G. (1998), Applied Production Analysis. A Dual Approach, Cambridge, Cambridge University Press.

Chianca, G.K. (2004), "A parceria entre a Embrapa e as organizações estaduais", Agroanalysis, vol. 24, N 5, Río de Janeiro.

Chomitz, K.M. y D.P. Gray (1996), "Roads, land markets, and deforestation: a spatial model of land use in Belize", The World Bank Economic Review, $\mathrm{N}^{\circ} 10$, Oxford, Oxford University Press.

Christensen, L.R. (1975), "Concepts and measurement of agricultural productivity", American Journal of Agricultural Economics, vol. 57, $\mathrm{N}^{\circ}$ 5, Oxford University Press.

Ciprandi, O. y J. Fert Neto (1996), "As perspectivas da pequena produção familiar na agricultura”, Ciência Rural, vol. 26, $\mathrm{N}^{\circ} 1$, Universidad Federal de Santa María.

Coelli, T.J. y D.S. Prasada Rao (2005), "Total factor productivity growth in agriculture: a Malmquist index analysis of 93 countries, 1980-2000", Agricultural Economics, vol. 32, N 1 , Asociación Internacional de Economistas Agrónomos. 
Coura, R.M., A.M. Figueiredo y M.L. Santos (2006), "Eficiência e tecnologia na agricultura paulista entre 1985 e 2001", Organizações Rurais \& Agroindustriais, vol. 8, $\mathrm{N}^{\circ} 2$.

Cropper, M., J. Puri y C. Griffiths (2001), "Predicting the location of deforestation: the role of roads and protected areas in north Thailand", Land Economics, vol. 77, $\mathrm{N}^{\circ} 2$, Madison, University of Wisconsin Press.

Davis, J.H. y R.A. Goldberg (1957), A Concept of Agribusiness, Boston, Universidad de Harvard.

Departamento de Agricultura de los Estados Unidos (2012), "Agricultural Productivity in the U.S.", Servicio de Investigación Económica [en línea] http://www.ers.usda.gov/ Data/AgProductivity.

(2008), Total Factor Productivity, Washington, D.C., Servicio de Investigación Económica.

Dimitri, C., A. Effland y N. Conklin (2005), "The 20th century transformation of U.S. agriculture and farm policy", Economic Information Bulletin, $\mathrm{N}^{\circ} 3$, Servicio de Investigación Económica.

FAO (Organización de las Naciones Unidas para la Agricultura y la Alimentación) (2012), "Production/Crops", Faostat [en línea] http://faostat.fao.org2012.

Fornazier, A. y J.E.R. Vieira Filho (2012), "Heterogeneidade estrutural no setor agropecuário brasileiro: evidências a partir do Censo Agropecuário de 2006", Texto para Discussão, $\mathrm{N}^{\circ} 1708$, Brasilia, Instituto de Investigación Económica Aplicada (IPEA).

Fuck, M.P. y M.B.M. Bonacelli (2007), "A necessidade de reorganização e de fortalecimento institucional do SNPA no Brasil", Revista de Política Agrícola, vol. 16, $\mathrm{N}^{\circ} 1$, Brasilia.

Fuglie, K.O. (2010), "Total factor productivity in the global agricultural economy: evidence from FAO data", The Shifting Patterns of Agricultural Production and Productivity Worldwide, J.M. Alston, B.A. Babcock y P.G. Pardey (eds.), Iowa, Universidad Estatal de Iowa.

Fuglie, K.O., J.M. MacDonald y E. Ball (2007), "Productivity growth in U.S. agriculture", Economic Brief, $\mathrm{N}^{\circ}$ 9, Departamento de Agricultura de los Estados Unidos/Servicio de Investigación Económica.

Fuglie, K.O., S.L. Wang y V.E. Ball (eds.) (2012), Productivity Growth in Agriculture: an International Perspective, Cambridge, Massachusetts, CABI.

Gasques, J.G. y otros (2014), "Produtividade da agricultura: resultados para o Brasil e estados selecionados", Revista Política Agrícola, vol. 23, $\mathrm{N}^{\circ} 3$.

(2013), Produtividade e crescimento: algumas comparações, Brasilia, Ministerio de Agricultura, Ganadería y Abastecimiento. (2012), "Produtividade da agricultura brasileira e os efeitos de algumas políticas", Brasilia, Ministerio de Agricultura, Ganadería y Abastecimiento, inédito.

(2010), "Produtividade total dos fatores e transformações da agricultura brasileira: análise dos dados dos censos agropecuários", A agricultura brasileira: desempenho, desafios e perspectivas, J.G. Gasques, J.E.R. Vieira Filho y Z. Navarro (orgs.), Brasilia, Instituto de Investigación Económica Aplicada (IPEA).

Gasques, J.G., E.T. Bastos y M.R.P. Bacchi (2008), "Produtividade e fontes de crescimento da agricultura brasileira", Políticas de incentivo à inovação tecnológica, J.A. de Negri y L.C. Kubota (eds.), Brasilia, Instituto de Investigación Económica Aplicada (IPEA).

(2007), "Produtividade e fontes de crescimento da agricultura", agosto [en línea] ftp://ftp.sp.gov.br/ftpiea/ congressos/cong-pal20807.pdf.

Gasques, J.G. y J.C.P.R. Conceição (2000), "Transformações estruturais da agricultura e produtividade total dos fatores", Texto para Discussão, $\mathrm{N}^{\circ} 768$, Brasilia, Instituto de Investigación Económica Aplicada (IPEA).
Gasques, J.G., J.E.R. Vieira Filho y Z. Navarro (orgs.) (2010), A agricultura brasileira: desempenho, desafios e perspectivas, Brasilia, Instituto de Investigación Económica Aplicada (IPEA).

Gasques, J.G., C.M. Villa Verde y J.A.F.G. Oliveira (2004), "Crédito rural e estruturas de financiamento. Brasília", Texto para Discussão, $\mathrm{N}^{\circ}$ 1036, Instituto de Investigación Económica Aplicada (IPEA).

Graziano da Silva, J.F. (1996), A nova dinâmica da agricultura brasileira, Campinas, Instituto de Economía/Universidad Estatal de Campinas (UNICAMP).

Hayami, Y. y V. Ruttan (1988), Desenvolvimento agrícola: teoria e experiências internacionais, Brasilia, Embrapa.

Hoffmann, R. (1980), Estatística para economistas, São Paulo, Pioneira.

IBGE (Instituto Brasileño de Geografía y Estadística) (2006), Censo Agropecuário 2006, Río de Janeiro.

Jorgenson, D.W. (1996), “The embodiment hypothesis", Productivity, vol. 1, Cambridge, Massachusetts, The MIT Press.

Ludena, C.E. (2010), "Agricultural productivity growth, efficiency change and technical progress in Latin America and the Caribbean", IDB Working Paper Series, $\mathrm{N}^{\circ} 186$, Washington, D.C., Banco Interamericano de Desarrollo (BID).

MAPA/AGE (Ministerio de Agricultura, Ganadería y Abastecimiento/ Asesoría de Gestión Estratégica) (2011), Produtividade total dos fatores, Brasilia.

Nohlen, D. y R. Sturm (1982), "La heterogeneidad estructural como concepto básico en la teoría de desarrollo", Revista de Estudios Políticos, $\mathrm{N}^{\circ} 28$.

OCDE (Organización para la Cooperación y el Desarrollo Económicos) (2011), Fostering Productivity and Competitiveness in Agriculture, París.

Paiva, R.M. (1971), "Modernização e dualismo tecnológico na agricultura", Pesquisa e Planejamento Econômico, vol. 1, № 2 , Instituto de Investigación Económica Aplicada.

Pinto, A. (1970), "Natureza e implicações da heterogeneidade estrutural da América Latina”, El Trimestre Económico, vol. 37, $\mathrm{N}^{\circ} 1$, Ciudad de México, Fondo de Cultura Económica.

Pires, J.F. y J.F. Garcia (2004), "Productivity of nations: a stochastic frontier approach to TFP decomposition", Texto para Discussão, $\mathrm{N}^{\circ}$ 143, São Paulo, Fundación Getulio Vargas.

Poudel, B.N., K.P. Paudel y D. Zilberman (2011), "Agricultural productivity convergence: myth or reality?", Journal of Agricultural and Applied Economics, vol. 43, $\mathrm{N}^{\circ} 1$.

Rodríguez, O. (1977), "Sobre la concepción del sistema centroperiferia", Revista de la CEPAL, $\mathrm{N}^{\circ} 3$, Santiago, Comisión Económica para América Latina y el Caribe (CEPAL).

Ruttan, V.W. (2002), "Productivity growth in world agriculture: sources and constraints", Journal of Economic Perspectives, vol. 16, $\mathrm{N}^{\circ} 4$, Nashville, Tennessee, American Economic Association.

Sadoulet, E. y A. de Janvry (1995), "Transactions costs and agrarian institutions", Quantitative Development Policy Analysis, Baltimore, The Johns Hopkins University Press.

Sunding, D. y D. Zilberman (2000), "The Agricultural Innovation Process: Research and Technology Adoption in a Changing Agricultural Sector" [en línea] http://are.berkeley.edu/ zilber11/ innovationchptr.pdf.

Sunkel, O. y R. Infante (orgs.) (2009), Hacia un desarrollo inclusivo: el caso de Chile (LC/L.3126), Santiago, Comisión Económica para América Latina y el Caribe (CEPAL).

Teixeira, E.C., F. Clemente y M.J.A. Braga (2013), “A contribuição das universidades para o desenvolvimento da agricultura no Brasil", Revista de Economia e Agronegócio, vol. 11, $\mathrm{N}^{\circ} 1$.

Thirtle, C. y P. Bottomley (1992), "Total factor productivity in UK agriculture, 1967-90", Journal of Agricultural Economics, vol. $43, \mathrm{~N}^{\circ} 3$, Wiley.

Vicente, J.R. (2003), "Tecnologia, eficiência e produtividade total de fatores na agricultura brasileira, 1970-95”, Sociedad Brasileña 
de Economía y Sociología Rural (SOBER) [en línea] http:// www.sober.org.br/palestra/2/633.pdf.

Vieira Filho, J.E.R. (2014), "Transformação histórica e padrões tecnológicos da agricultura brasileira", $O$ mundo rural do Brasil no século 21: a formação de um novo padrão agrário e agrícola, A.M. Buainain y otros (orgs.), Brasilia, Embrapa.

(2013), "Heterogeneidad estructural de la agricultura familiar en el Brasil", Revista CEPAL, N 111 (LC/G.2597-P), Santiago, Comisión Económica para América Latina y el Caribe (CEPAL)

(2012), "Technological trajectories and learning in agricultural sector", 10th GLOBELICS International Conference.

(2009), "Inovação tecnológica e aprendizado agrícola: uma abordagem schumpeteriana", tesis, Campinas, Instituto de Economía/Universidad Estatal de Campinas.

Vieira Filho, J.E.R. y J.M.F. Silveira (2012), "Mudança tecnológica na agricultura: uma revisão crítica da literatura e o papel das economias de aprendizado", Revista de Economia e Sociologia Rural, vol. 50, N 4, Brasilia, Sociedad Brasileña de Economía y Sociología Rural.
(2011), "Modelo evolucionário de aprendizado agrícola", Revista Brasileira de Inovação, vol. 10, $\mathrm{N}^{\circ} 4$, Campinas.

Vieira Filho, J.E.R., A.C. Campos y C.M.C. Ferreira (2005), "Abordagem alternativa do crescimento agrícola: um modelo de dinâmica evolucionária", Revista Brasileira de Inovação, vol. 4, $\mathrm{N}^{\circ}$ 2, Campinas.

Vieira Filho, J.E.R., G.R. Santos y A. Fornazier (2013), "Distribuição produtiva e tecnológica da agricultura brasileira e sua heterogeneidade estrutural", Texto para Discussão, $\mathrm{N}^{\circ} 54$ (LC/BRS/R.277), Brasilia, Comisión Económica para América Latina y el Caribe (CEPAL)/Instituto de Investigación Económica Aplicada (IPEA).

Wilkinson, J. (2010), "Transformações e perspectivas dos agronegócios brasileiros", Revista Brasileira de Zootecnia, vol. 39, Sociedad Brasileña de Zootecnia.

Yokoyama, L.P. y A.C.M. Igreja (1992), "Principais lavouras da região centro-oeste: variações no período 1975-1987”, Pesquisa Agropecuária Brasileira, vol. 27, ํㅜ 5. 